\title{
Circular Scale of Time and Its Use in Calculating the Schrödinger Perturbation Energy of a Non-Degenerate Quantum State
}

\author{
Stanisław Olszewski \\ Institute of Physical Chemistry, Polish Academy of Sciences, Warsaw, Poland \\ Email: solszewski@ichf.edu.plolsz@ichf.edu.pl
}

How to cite this paper: Olszewski, S. (2020) Circular Scale of Time and Its Use in Calculating the Schrödinger Perturbation Energy of a Non-Degenerate Quantum State. Journal of Modern Physics, 11, 1536-1558.

https://doi.org/10.4236/jmp.2020.1110095

Received: July 20, 2020

Accepted: October 13, 2020

Published: October 16, 2020

Copyright $\odot 2020$ by author(s) and Scientific Research Publishing Inc. This work is licensed under the Creative Commons Attribution International License (CC BY 4.0). http://creativecommons.org/licenses/by/4.0/

\begin{abstract}
The paper presents a circular scale of time-and its diagrams-which can be successfully applied in calculating the Schrödinger perturbation energy of a non-degenerate quantum state. This seems to be done in a more simple way than with the aid of any other of the perturbation approaches of a similar kind. As an example of the theory suitable to comparison is considered the Feynman diagrammatic method based on a straight-linear scale of time which represents a much more complicated formalism than the present one. All diagrams of the approach outlined in the paper can obtain as their counterparts the algebraic formulae which can be easily extended to an arbitrary Schrödinger perturbation order. The calculations and results descending from the perturbation orders $N$ between $N=1$ and $N=7$ are reported in detail.
\end{abstract}

\section{Keywords}

Circular Scale of Time, Schrödinger Perturbation Energy in Non-Relativistic Quantum Mechanics, Non-Degenerate Quantum States

\section{Introduction}

What is time? My answer is that it is a parameter which allows us to distinguish a later event from an earlier one; this distinction seems to be a fundamental property of time. On the other hand, according to Springer's "Physikalisches Handwörterbuch" [1], time is defined as an independent variable of classical mechanics. One is suggested to add here the adjective "non-relativistic" to the notion of mechanics, because the relativity-in its special picture-makes any time interval dependent on such parameters as the body velocity and light velocity. Evidently in the general relativity the dependence of time is still more ex- 
tended, for example due to the presence of the mass of the body [2].

In science an important problem of time became to couple its behaviour with some other physical properties than those given by classical mechanics. Perhaps the best known example is here the entropy and its connections with time. In brief we need the parameters, or effects, which can be examined parallelly with time, though they do not necessarily represent an explicit dependence on the time variable.

In the present case such example of the time connected with physics is given by a quantum perturbation effect. We assume that at some time moment-more or less accurately known-some time-independent perturbation to a quantum system is applied. For a ground state in the absence of the perturbation effect the notion of a stationary state implies an infinite duration of that state. Usually we are unable to follow in detail the history of a system changed by the perturbation, but-according to Schrödinger-we know the end of the state history equivalent to the end of the perturbation process: this is a new stationary state having a new eigenenergy, different than possessed by the system state before the perturbation was applied.

Our aim is to present the time dependence of the perturbation history-and its results-in a possibly transparent way.

\section{Quantum-Mechanical Characteristics of the Schrödinger Perturbation Process}

In fact the original characteristics of the perturbation process done by Schrödinger [3] did not involve the idea, or a variable, of time. Also in more modern treatments of the Schrödinger perturbation theory-see e.g. [4] - the time does not enter the calculations.

In fact when the Hamiltonian operator $\hat{H}_{0}$ of an unperturbed quantum problem is given, the main idea is to calculate the eigenenergies $E^{(0)}$ and eigenfunctions $\psi^{(0)}$ satisfying the eigenequation

$$
\hat{H}^{(0)} \psi^{(0)}(\boldsymbol{r})=E^{(0)} \psi^{(0)}(\boldsymbol{r})
$$

The $E^{(0)}$ are constant energy terms and $\psi^{(0)}(\boldsymbol{r})$ are eigenfunctions dependent solely on the position vector parameter $\boldsymbol{r}$. In principle there can exist an infinite set of $\psi^{(0)}(\boldsymbol{r})$ and $E^{(0)}$.

Let the perturbed problem be due to introduction of the so-called small perturbation potential

$$
V^{\text {per }}=V^{\text {per }}(r)
$$

which is dependent only on the position variable $\boldsymbol{r}$. By assuming-for the sake of simplicity - that the unperturbed problem is a non-degenerate one, we look now for the solution of the perturbed eigenequation

$$
\hat{H}^{\text {per }} \psi^{\text {per }}(\boldsymbol{r})=\left[\hat{H}^{(0)}+V^{\text {per }}(\boldsymbol{r})\right] \psi^{\text {per }}(\boldsymbol{r})=E^{\text {per }} \psi^{\text {per }}(\boldsymbol{r})
$$

where 


$$
E^{\text {per }} \text { and } \psi^{\text {per }}(\boldsymbol{r})
$$

are the sets of the energy eigenvalues and eigenfunctions calculated respectively to some chosen perturbation potential (2).

The idea of Schrödinger and his followers became-instead of solving (3) - to calculate the perturbed quantities (4) in terms of the sets of unperturbed $E_{n}^{(0)}$ and $\psi_{n}^{(0)}(\boldsymbol{r})$ belonging to various solutions $n$. If we limit the calculations to the energy problem alone, the perturbed term for energy can be represented by a series of terms belonging to different perturbation orders $N$, where $N$ varies according to the sequence of the integer numbers

$$
N=1,2,3,4, \cdots, N_{\max } \text {. }
$$

The order $N$ can be referred to the perturbation energy $E^{\text {per }}$ and perturbation wave function $\psi^{\text {per }}(\boldsymbol{r})$ of a non-degenerate quantum state by the formulae (see e.g. [4]):

$$
E^{\text {per }}=E^{(0)}+\lambda \Delta E_{1}+\lambda^{2} \Delta E_{2}+\lambda^{3} \Delta E_{3}+\cdots+\lambda^{N_{\max }} \Delta E_{N_{\max }}
$$

whereas

$$
\psi^{\text {per }}=\psi^{(0)}+\lambda \Delta \psi^{(1)}+\lambda^{2} \Delta \psi^{(2)}+\lambda^{3} \Delta \psi^{(3)}+\cdots+\lambda^{N_{\max }} \Delta \psi^{\left(N_{\max }\right)} .
$$

The both series, (5a) and (5b), are expressed in terms of the powers of a parameter $\lambda$. These powers of $\lambda$ represent in (5a) the order given in (5) of the energy correction $\Delta E_{N}$ and in (5b) the order of the wave function correction $\Delta \psi^{(N)}$, respectively.

The number $N_{\max }$ denotes a maximal value of $N$ applied in some practical calculation. In effect, for a convergent perturbation method and $N_{\max }$ sufficiently large, the accuracy of results for the perturbed energy is expected to increase with the increase of a chosen $N_{\max }$. In many occasions-in order to get a good approximation of the perturbed energy $E^{\text {per }}$-there is necessary to calculate a series of terms due to a large $N_{\max }$ :

$$
E^{\mathrm{per}}=E^{(0)}+\Delta E^{(1)}+\Delta E^{(2)}+\Delta E^{(3)}+\cdots .
$$

Here we have put $\lambda=1$ in (5a) and the term $E^{(0)}$-entering also (5a)-labels the energy of an unperturbed state. The superscript entering $\Delta E$ represents the energy contribution due to the energy contribution to (6) due to the perturbation order $N$.

The subscript $n$ which labels the index of the quantum state submitted to perturbation has been omitted in (5a), (5b) and (6) for the sake of brevity. Also, for the same reason, $N_{\max }$ entering further calculations will be replaced simply by $N$.

The main — and a rather fundamental problem of the Schrödinger theory-is that the number and complication of the perturbation terms which are necessary for calculating any

$$
\Delta E^{(N)}
$$

entering (6) increases rapidly with $N$; an increase of the number of terms neces- 
sary to obtain (7) is represented by the formula [5] [6]

$$
S_{N}=\frac{(2 N-2) !}{N !(N-1) !}
$$

and detailed values of $S_{N}$ are given in Table 1 . But simultaneously-to the best of my knowledge - no systematic rule was provided to build up the set of individual terms entering (8), and this task becomes a much complicated one for large $N$.

In effect the calculation of terms (7) suitable for large $N$ becomes a difficult task already at the stage of their construction. But a removal of this complication provides us not only with a simplicity necessary to solve the calculational problem. In fact, the importance of the perturbation methods in general can be considered as a decreasing obstacle in view of the development of the computational machinery and its technique applied to solve the physical problems. The point of importance is that an essential simplification can be attained due to the introduction of the time parameter into the perturbation theory. This introduction provides us with a suitable arrangement of the time points on the scale labelling the contact events of the perturbation potential with an originally unperturbed quantum system. The details of this idea and its use in the Schrödinger method are presented below.

\section{Perturbation Order and a Suitable Scale of Time}

Not only in the everyday life, but in physics too, we are accustomed to applying a straight-linear scale of time according to which each of the later events does happen after an earlier one. Topologically the scale does assume the shape of an infinite straight line on which a distance of some chosen earlier point to an actual point of time increases systematically with the time variable; see Figure 1. This

Table 1. The $S_{N}$ numbers from formula (8) and Feynman's $P(N)$ numbers of formula (9) (see [8]) calculated for different $N$.

\begin{tabular}{ccc}
\hline Perturbation order $N$ & $S(N)$ & $P(N)$ \\
\hline 1 & 1 & 1 \\
2 & 1 & 1 \\
3 & 2 & 2 \\
4 & 5 & 6 \\
5 & 14 & 24 \\
6 & 42 & 120 \\
7 & 132 & 720 \\
8 & 429 & 5040 \\
9 & 1430 & 40,320 \\
10 & 4862 & 362,880 \\
11 & 16,796 & $3,628,800$ \\
12 & 58,786 & $39,916,800$
\end{tabular}




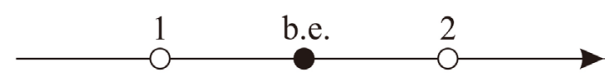

Figure 1. The straight-linear (progressive) scale of time. The point b.e. represents the present situation: $b$ is the beginning point of the future (on the right), the point $e$ is the end point of the past (on the left). An access is solely from point 1 to 2 ; no access is from 2 to 1 .

situation does not change also in the case when-according to the Feynman's idea-the terms of the Schrodinger perturbation theory are sought to be plotted along the scale with the aim to calculate the necessary diagrams of energy [7] [8]. These diagrams can be classified also according to the perturbation order $N$, however, in order to get a contribution of energy $\Delta E^{(N)}$ given in (7) the number of diagrams should be not that presented in (8) but becomes

$$
P(N)=(N-1) \text { ! }
$$

Only for very small $N$ we have

$$
P(N)=S(N)
$$

but for $N \gg 1$ the inequality

$$
P(N) \gg S(N)
$$

evidently does exist giving for example for $N=20$ the ratio

$$
P(20): S(20) \cong 0.7 \times 10^{8},
$$

The formulae (11) and (12) imply that in order to get-in average-a single Schrödinger component term for the perturbation energy of a non-degenerate quantum state-a large, or even very large, number of results due to the Feynman energy diagrams should be first calculated, next suitably combined. Such a difficulty does not apply to the calculations based on a circular scale developed in the present paper.

\section{Perturbation Process along a Circular Scale of Time and Its Energy Terms}

We assume that the perturbation process is a set of successive collisions of the perturbation potential (2) with an unperturbed quantum system. The collision events are extended along a topological circle characteristic for a given order $N$ of the perturbation potential; in the next step the collisions are labelled by separate time points whose number is equal to $N$. Therefore the number of the time points on the scale increases gradually with the increase of $N$; see Figure 2 and Figure 3.

A characteristic feature is that the set of the time points present on the scale characteristic for a given $N$ is sufficient to represent all $S_{N}$ perturbation terms given in (8); moreover we obtain a one-to-one correspondence between the individual diagrams obtained with the aid of the scale and the Schrödinger energy terms entering the perturbation order $N$; see [9] [10] [11]. This goal can be attained on condition the following rules concerning formation of supplementary diagrams characteristic for any $N$ are satisfied: 


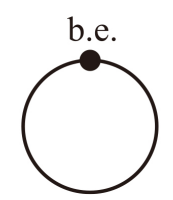

Figure 2. Time scale for the perturbation order $N=1$. Only one time point (beginning-end = b.e.) is present on the scale.

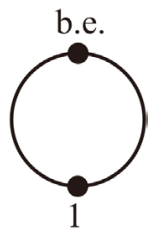

Figure 3. Time scale for the perturbation order $N=2$. Beyond the beginning-end (b.e.) point there is only one other point 1 on the scale.

1) one of the time points on each scale is considered as the beginning-end point of that scale and this point cannot be submitted to contractions with the other time points present on that scale;

2) the lines created in result of contractions of the time points on the scale should not cross;

3) any other contraction of the time points than that satisfying the rules 1) and 2) should not be taken into account.

In effect, beyond the time loops indicated in Figure 1 and Figure 3 also other loops of time can be created; they correspond to $N>2$ and are discussed below. In the terminology applied henceforth the time loop having the beginning-end point on it is called the main loop of time; it is a single loop on any diagram. The other loops of time, called the side loops, are due to contraction, or contractions, of the time points; see Section 6.

A general look on the time-point contractions and their applications is given in Section 10.

\section{Energy Terms Belonging to $N=1$ and $N=2$}

Evidently-according to the rules 1) and 2) given above-no contraction as well as no side loop can be created for $N=1$ and $N=2$. The first contraction of the time points is possible for $N=3$ between the points 1 and 2 represented by the formula

$$
t_{1}: t_{2}=1: 2
$$

In this case, beyond a non-contracted diagram for $N=3$ presented in Figure 4, we obtain a new diagram connected with (13); see Figure 5. In effect we obtain for $N=3$ the number of two diagrams: that of Figure 4 and that of Figure 5 . This is in accordance with the formula (8) from which we have

$$
S_{3}=2 \text {. }
$$




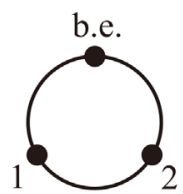

Figure 4. Time scale for the perturbation order $N=3$. Beyond the beginning-end (b.e.) point, there are two other points, 1 and 2, on the scale.

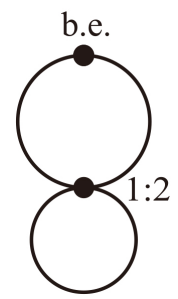

Figure 5. Contraction of the time points 1 and 2 present on the scale representing the perturbation order $N=3$ creates a side loop of time on the scale similar to the time loop characteristic for $N=1$; see Figure 2.

It is easy to check that

$$
S_{1}=S_{2}=1
$$

which imply only single diagrams present for $N=1$ and $N=2$ in Figure 1 and Figure 3 respectively. Let us consider now the energy terms associated with the obtained diagrams.

The perturbation energy connected with $N=1$ is represented by

$$
\Delta E_{1}=\langle V\rangle=\left\langle n\left|V^{\text {per }}\right| n\right\rangle
$$

which is a single matrix element.

On the other hand, for $N=2$ a summation process over the running states $p$ different than $n$ is involved:

$$
\Delta E_{2}=\langle V P V\rangle=\sum_{p \neq n} \frac{\left\langle n\left|V^{\text {per }}\right| p\right\rangle\left\langle p\left|V^{\text {per }}\right| n\right\rangle}{E_{n}^{(0)}-E_{p}^{(0)}} .
$$

The symbols $V$ are connected with the matrix elements in the numerator, symbol $P$ refers to a single energy difference in the denominator.

\section{Contractions of the Time Points on the Scale Provide us with the Side Loops of Time; Perturbation Orders $N=3$ and $N=4$}

For $N=3$ we have three time points on the scale: 1,2 , and 3 . Let 3 be the beginning-end point, so the points 1 and 2 can be submitted to contraction:

$$
t_{1}: t_{2}=1: 2
$$

no contraction with point 3 can be applied. Since $S_{3}=2$ we have two Schrödinger terms for $N=3$. The first one corresponds to the lack of contractions on the 
scale; see Figure 4 . This gives the energy term

$$
\langle V P V P V\rangle=\sum_{p} \sum_{q} \frac{\left\langle n\left|V^{\mathrm{per}}\right| p\right\rangle\left\langle p\left|V^{\mathrm{per}}\right| q\right\rangle\left\langle q\left|V^{\mathrm{per}}\right| n\right\rangle}{\left(E_{n}^{(0)}-E_{p}^{(0)}\right)\left(E_{n}^{(0)}-E_{q}^{(0)}\right)}
$$

where $p \neq n$ and $q \neq n$. On the other side, the contraction (18) (see Figure 5) gives the energy term

$$
-\left\langle V P^{2} V\right\rangle\langle V\rangle
$$

which is a product of

$$
\left\langle V P^{2} V\right\rangle=\sum_{p \neq n} \frac{\left\langle n\left|V^{\mathrm{per}}\right| p\right\rangle\left\langle p\left|V^{\mathrm{per}}\right| n\right\rangle}{\left(E_{n}^{(0)}-E_{p}^{(0)}\right)^{2}}
$$

and $\langle V\rangle$ which is the term given in (16). The product (20) is taken with a minus sign.

It has to be noted that the power of the energy term in the denominator in (21) is equal to the power of $P$ on the left of (21). The minus sign in (20) is dictated by the even number of the bracket terms present in the product in (20); an odd number of the bracket terms presenting an energy term leads to a plus sign for that term; see (16), (17) and (19).

The term $\langle V\rangle$ in (20) represents a contribution due to a side loop of time created by contraction (18); see Figure 5 . Because of a difference of the time point indices 2 and 1 entering (18) which is equal to

$$
2-1=1 \text {, }
$$

the side loop created by contraction (18) contributes the term

$$
\langle V\rangle=\Delta E_{1}
$$

entering as a multiplier in (20). In effect the total perturbation energy of $N=3$ is equal to a sum:

$$
\Delta E_{3}=\langle V P V P V\rangle-\left\langle V P^{2} V\right\rangle \Delta E_{1}
$$

because of (23) taken into account in (20).

The energy belonging to the order $N=4$ (see Figure 6) can be considered in a similar way. If the beginning-end point on the scale is labelled by 4 , we have three time points

$$
t_{1}=1, t_{2}=2, t_{3}=3,
$$

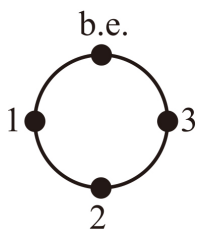

Figure 6. Time scale for the perturbation order $N=4$. Beyond the beginning-end (b.e.) point, three other points (1,2 and 3$)$ are present on the scale. 
which are suitable to contractions. Without any time contraction the contribution to energy is represented by the term

$$
\langle V P V P V P V\rangle=\sum_{p} \sum_{q} \sum_{r} \frac{\left\langle n\left|V^{\mathrm{per}}\right| p\right\rangle\left\langle p\left|V^{\mathrm{per}}\right| q\right\rangle\left\langle q\left|V^{\mathrm{per}}\right| r\right\rangle\left\langle r\left|V^{\mathrm{per}}\right| n\right\rangle}{\left(E_{n}^{(0)}-E_{p}^{(0)}\right)\left(E_{n}^{(0)}-E_{q}^{(0)}\right)\left(E_{n}^{(0)}-E_{r}^{(0)}\right)}
$$

where $p, q, r \neq n$. Next come contractions of the points in (25):

$$
\begin{aligned}
t_{1}: t_{2} & =1: 2, \\
t_{1}: t_{3} & =1: 3, \\
t_{2}: t_{3} & =2: 3, \\
t_{1}: t_{2}: t_{3} & =1: 2: 3 .
\end{aligned}
$$

The contractions presented in (27)-(30) give respectively the energy terms:

$$
-\left\langle V P^{2} V P V\right\rangle\langle V\rangle=-\sum_{p} \sum_{q} \frac{\left\langle n\left|V^{\mathrm{per}}\right| p\right\rangle\left\langle p\left|V^{\mathrm{per}}\right| q\right\rangle\left\langle q\left|V^{\mathrm{per}}\right| n\right\rangle}{\left(E_{n}^{(0)}-E_{p}^{(0)}\right)^{2}\left(E_{n}^{(0)}-E_{q}^{(0)}\right)}\langle V\rangle
$$

where $p, q \neq n$ and $\langle V\rangle=\Delta E_{1} \quad$ [contraction 1:2],

$$
-\left\langle V P^{2} V\right\rangle\langle V P V\rangle=-\sum_{p} \frac{\left\langle n\left|V^{\mathrm{per}}\right| p\right\rangle\left\langle p\left|V^{\mathrm{per}}\right| n\right\rangle}{\left(E_{n}^{(0)}-E_{p}^{(0)}\right)^{2}} \sum_{q} \frac{\left\langle n\left|V^{\mathrm{per}}\right| q\right\rangle\left\langle q\left|V^{\mathrm{per}}\right| n\right\rangle}{E_{n}^{(0)}-E_{q}^{(0)}}
$$

where $p, q \neq n$ and $\langle V P V\rangle=\Delta E_{2}$ because of (17) [contraction 1:3],

$$
-\left\langle V P V P^{2} V\right\rangle\langle V\rangle=-\sum_{p} \sum_{q} \frac{\left\langle n\left|V^{\mathrm{per}}\right| p\right\rangle\left\langle p\left|V^{\mathrm{per}}\right| q\right\rangle\left\langle q\left|V^{\mathrm{per}}\right| n\right\rangle}{\left(E_{n}^{(0)}-E_{p}^{(0)}\right)\left(E_{n}^{(0)}-E_{q}^{(0)}\right)^{2}}\langle V\rangle
$$

where $p, q \neq n$ and $\langle V\rangle=\Delta E_{1}$ [contraction 2:3],

$$
\left\langle V P^{3} V\right\rangle(\langle V\rangle)^{2}=\sum_{p} \frac{\left\langle n\left|V^{\mathrm{per}}\right| p\right\rangle\left\langle p\left|V^{\mathrm{per}}\right| n\right\rangle}{\left(E_{n}^{(0)}-E_{p}^{(0)}\right)^{3}}(\langle V\rangle)^{2}
$$

where $p \neq n$ and $(\langle V\rangle)^{2}=\left(\Delta E_{1}\right)^{2}$ [contraction 1:2:3]. Let us note that the sum of powers of $P$ in any energy term is equal to $N-1=3$, and the sum of powers of $V$ within the brackets of each energy term is $N=4$.

Together with the energy term (26) we obtain from (31)-(34)

$$
S_{4}=\frac{(2 \times 4-2) !}{3 ! 4 !}=\frac{6 !}{3 ! 4 !}=5
$$

energy terms for $N=4$. The perturbation energy belonging to $N=4$ is equal to a sum of five terms given in (26) and (31)-(34):

$$
\begin{aligned}
\Delta E_{4}= & \langle V P V P V P V\rangle-\left\langle V P^{2} V P V\right\rangle \Delta E_{1}-\left\langle V P^{2} V\right\rangle \Delta E_{2} \\
& -\left\langle V P V P^{2} V\right\rangle \Delta E_{1}+\left\langle V P^{3} V\right\rangle\left(\Delta E_{1}\right)^{2} .
\end{aligned}
$$

Evidently the fourth term on the right of (36) is equal to the second term because of symmetry.

The rule defining the sign of the perturbation terms is very simple: for an odd number of terms entering the product giving a perturbation term the sign of 
product is positive; an even number of terms entering a similar product giving a perturbation term makes this term to have a negative sign.

\section{Energy of the Perturbation Orders $N=5$ and $N=6$}

The time scales corresponding to above $N$ are presented in Figure 7 and Figure 8.

Here the recurrence procedure can be useful to apply, so for $N=5$ we take first into account the perturbation terms of the order $N$ lower than 5, and for $N$ $=6$ the terms of the order lower than 6 , respectively.

In this way the first five terms belonging to the order $N=5$ can be obtained from $S_{4}=5$ terms of Section 6 by introducing the time point 4 as a free point different than the beginning-end point of time. This makes any bracket contribution due to the main loop of time entering $\Delta E_{4}$ [see (36)] changed by an increase equal to $P V$ put at the end of the bracket term. The first 5 energy terms belonging to $\Delta E_{5}$ are:

$$
\begin{aligned}
& \langle V P V P V P V P V\rangle-\left\langle V P^{2} V P V P V\right\rangle \Delta E_{1}-\left\langle V P^{2} V P V\right\rangle \Delta E_{2} \\
& -\left\langle V P V P^{2} V P V\right\rangle \Delta E_{1}+\left\langle V P^{3} V P V\right\rangle\left(\Delta E_{1}\right)^{2} .
\end{aligned}
$$

The first term in (37) is a modification of the term (26), the remaining four energy terms in (37) are due to suitable modifications of the terms entering (36).

Further contributions to $\Delta E_{5}$ are due to the fact that in the case of $N=5$ the new time point 4 can be submitted also to contractions. They begin with point 1 and the other points between 1 and 4 :

$$
1: 4,1: 2: 4,1: 3: 4,1: 2: 3: 4 \text {. }
$$

The contractions in (38) together with the side loops created by them give the following energy terms:

$$
-\left\langle V P^{2} V\right\rangle \Delta E_{3},\left\langle V P^{3} V\right\rangle \Delta E_{1} \Delta E_{2},\left\langle V P^{3} V\right\rangle \Delta E_{2} \Delta E_{1},-\left\langle V P^{4} V\right\rangle\left(\Delta E_{1}\right)^{3} .
$$

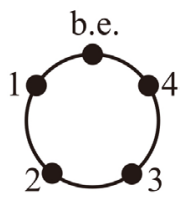

Figure 7. Time scale for the perturbation order $N=5$. Beyond the beginning-end (b.e.) point, four other points $(1,2,3$ and 4$)$ are present on the scale.

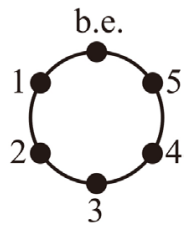

Figure 8. Time scale for the perturbation order $N=6$. Beyond the beginning-end (b.e.) point, five other points $(1,2,3,4$ and 5$)$ are present on the scale. 
Since $\Delta E_{3}$ present in the first term in (39) gives two Schrödinger perturbation terms, the set of terms in (39) represents the next $S_{4}=5$ perturbation terms of energy belonging to $N=5$. The index 4 of $S_{4}$ refers to a maximal number 4 of the time points entering contractions (38).

In a further step contraction of the time points 2 and 3 with point 4 have to be considered. They are

$$
2: 4,2: 3: 4
$$

which give respectively two perturbation energy terms:

$$
-\left\langle V P V P^{2} V\right\rangle \Delta E_{2},\left\langle V P V P^{3} V\right\rangle\left(\Delta E_{1}\right)^{2} \text {. }
$$

The last set of the energy perturbation terms belonging to $N=5$ is given by a single contraction

$$
3: 4 \text {. }
$$

In this case the time points 1 and 2 present before point 3 can be either free, or contracted together. For 1 and 2 free the contraction in (42) gives the perturbation term

$$
-\left\langle V P V P V P^{2} V\right\rangle \Delta E_{1}
$$

On the other hand, the contraction 1:2 combined with that in (42) gives the perturbation term

$$
1: 2 \bigcap 3: 4 \rightarrow\left\langle V P^{2} V P^{2} V\right\rangle\left(\Delta E_{1}\right)^{2} .
$$

In effect we obtain from (37), (39), (41), (43) and (44) a sum of

$$
S_{5}=\frac{(2 \times 5-2) !}{4 ! 5 !}=\frac{8 !}{4 ! 5 !}=14
$$

perturbation terms belonging to $N=5$, if we note that $\Delta E_{3}$ in (39) combines two Schrödinger perturbation terms.

A full perturbation energy of the order $N=5$ becomes a sum of $S_{5}$ terms entering the formulae quoted before (45):

$$
\begin{aligned}
\Delta E_{5}= & \langle V P V P V P V P V\rangle-\left\langle V P^{2} V P V P V\right\rangle \Delta E_{1}-\left\langle V P^{2} V P V\right\rangle \Delta E_{2} \\
& -\left\langle V P V P^{2} V P V\right\rangle \Delta E_{1}+\left\langle V P^{3} V P V\right\rangle\left(\Delta E_{1}\right)^{2}-\left\langle V P^{2} V\right\rangle \Delta E_{3} \\
& +\left\langle V P^{3} V\right\rangle \Delta E_{1} \Delta E_{2}+\left\langle V P^{3} V\right\rangle \Delta E_{2} \Delta E_{1}-\left\langle V P^{4} V\right\rangle\left(\Delta E_{1}\right)^{3} \\
& -\left\langle V P V P^{2} V\right\rangle \Delta E_{2}+\left\langle V P V P^{3} V\right\rangle\left(\Delta E_{1}\right)^{2} \\
& -\left\langle V P V P V P^{2} V\right\rangle \Delta E_{1}+\left\langle V P^{2} V P^{2} V\right\rangle\left(\Delta E_{1}\right)^{2} .
\end{aligned}
$$

Again, because of the presence of $\Delta E_{3}$, the sixth term on the right of (46) represents two Schrödinger perturbation terms. Evidently-because of symmetry-some terms entering (46), for example the second term and one-by-last term on the right, become equal.

The calculation of $\Delta E_{6}$ being the energy of the perturbation order $N=6$ is much similar. The first 14 terms are obtainable from the energy expression (46) 
representing $\Delta E_{5}$ due to the fact of supplying the time point 5 as a free point for the case of $N=6$. The corresponding part of the perturbation energy $\Delta E_{6}$ comes by adding the $P V$ term at the end of any bracket term in $\Delta E_{5}$ which is due to the main loop of time. On the basis of (46) we obtain the following contribution of $S_{5}=14$ energy terms entering $\Delta E_{6}$ :

$$
\begin{aligned}
& \langle V P V P V P V P V P V\rangle-\left\langle V P^{2} V P V P V P V\right\rangle \Delta E_{1}-\left\langle V P^{2} V P V P V\right\rangle \Delta E_{2} \\
& -\left\langle V P V P^{2} V P V P V\right\rangle \Delta E_{1}+\left\langle V P^{3} V P V P V\right\rangle\left(\Delta E_{1}\right)^{2}-\left\langle V P^{2} V P V\right\rangle \Delta E_{3} \\
& +\left\langle V P^{3} V P V\right\rangle \Delta E_{1} \Delta E_{2}+\left\langle V P^{3} V P V\right\rangle \Delta E_{2} \Delta E_{1}-\left\langle V P^{4} V P V\right\rangle\left(\Delta E_{1}\right)^{3} \\
& -\left\langle V P V P^{2} V P V\right\rangle \Delta E_{2}+\left\langle V P V P^{3} V P V\right\rangle\left(\Delta E_{1}\right)^{2}-\left\langle V P V P V P^{2} V P V\right\rangle \Delta E_{1} \\
& +\left\langle V P^{2} V P^{2} V P V\right\rangle\left(\Delta E_{1}\right)^{2} .
\end{aligned}
$$

In the next step we take into account that the time point 5 for $N=6$ can contract with point 1 and all points between 1 and 5 . This gives the following contractions and the energy terms corresponding to them:

$$
\begin{aligned}
& 1: 5 \rightarrow-\left\langle V P^{2} V\right\rangle \Delta E_{4} \\
& 1: 2: 5 \rightarrow\left\langle V P^{3} V\right\rangle \Delta E_{1} \Delta E_{3} \\
& 1: 3: 5 \rightarrow\left\langle V P^{3} V\right\rangle\left(\Delta E_{2}\right)^{2} \\
& 1: 4: 5 \rightarrow\left\langle V P^{3} V\right\rangle \Delta E_{3} \Delta E_{1} \\
& 1: 2: 3: 5 \rightarrow-\left\langle V P^{4} V\right\rangle\left(\Delta E_{1}\right)^{2} \Delta E_{2} \\
& 1: 2: 4: 5 \rightarrow-\left\langle V P^{4} V\right\rangle \Delta E_{1} \Delta E_{2} \Delta E_{1} \\
& 1: 3: 4: 5 \rightarrow-\left\langle V P^{4} V\right\rangle \Delta E_{2}\left(\Delta E_{1}\right)^{2} \\
& 1: 2: 3: 4: 5 \rightarrow\left\langle V P^{5} V\right\rangle\left(\Delta E_{1}\right)^{4}
\end{aligned}
$$

In the brackets at the end of each row is given the number of Schrödinger energy terms connected with the considered row. This means that (48)-(55) give next $S_{5}=14$ Schrödinger perturbation terms. Here 5 is a maximal number of points entering contractions listed in the above formulas. The other time contractions are:

$$
\begin{aligned}
& 2: 5 \rightarrow-\left\langle V P V P^{2} V\right\rangle \Delta E_{3} \\
& 2: 3: 5 \rightarrow\left\langle V P V P^{3} V\right\rangle \Delta E_{1} \Delta E_{2} \\
& 2: 4: 5 \rightarrow\left\langle V P V P^{3} V\right\rangle \Delta E_{2} \Delta E_{1} \\
& 2: 3: 4: 5 \rightarrow-\left\langle V P V P^{4} V\right\rangle\left(\Delta E_{1}\right)^{3}
\end{aligned}
$$

with the time point 1 left free giving 5 perturbation terms because the index 4 due to presence of contraction points gives $S_{4}=5$.

But both points 1 and 2 can be left free combining with contractions 


$$
\begin{gathered}
3: 5 \rightarrow-\left\langle V P V P V P V P^{2} V\right\rangle \Delta E_{2}, \\
3: 4: 5 \rightarrow\left\langle V P V P V P V P^{3} V\right\rangle\left(\Delta E_{1}\right)^{2} .
\end{gathered}
$$

Another situation is obtained when 1 and 2 contract together, in this case we obtain in place of (60) and (61) the energy terms combined with 1:2, so

$$
\begin{gathered}
1: 2 \cap 3: 5 \rightarrow\left\langle V P^{2} V P^{2} V\right\rangle \Delta E_{1} \Delta E_{2}, \\
1: 2 \cap 3: 4: 5 \rightarrow-\left\langle V P^{2} V P^{3} V\right\rangle\left(\Delta E_{1}\right)^{3} .
\end{gathered}
$$

In effect from the formulae (60) to (61a) we obtain next 4 perturbation terms belonging to $\Delta E_{6}$.

Finally a single contraction

$$
4: 5
$$

can combine either with the free points

$$
1,2,3
$$

giving one energy term

$$
-\left\langle V P V P V P V P^{2} V\right\rangle \Delta E_{1},
$$

or with contractions of 1,2 , and 3, viz.

$$
\begin{gathered}
1: 2 \cap 4: 5 \rightarrow\left\langle V P^{2} V P V P^{2} V\right\rangle\left(\Delta E_{1}\right)^{2}, \\
1: 3 \cap 4: 5 \rightarrow\left\langle V P^{2} V P^{2} V\right\rangle \Delta E_{2} \Delta E_{1}, \\
1: 2: 3 \cap 4: 5 \rightarrow-\left\langle V P^{3} V P^{2} V\right\rangle\left(\Delta E_{1}\right)^{3}, \\
2: 3 \cap 4: 5 \rightarrow\left\langle V P V P^{2} V P^{2} V\right\rangle\left(\Delta E_{1}\right)^{2},
\end{gathered}
$$

which give together four energy terms presented in the second step of (65)-(68).

In effect the number of the perturbation terms belonging to $N=6$ obtained from (47), (48)-(55), (56)-(59), (60)-(61a), and (62)-(68) becomes:

$$
14+14+5+4+1+4=42=S_{6}
$$

which is the expected result; see Table 1 . A full perturbation energy of the order $N=6$ is equal to a sum of the terms belonging to expressions listed above equation (69); see (47)-(61a) and (64)-(68).

\section{Perturbation Energy Belonging to $N=7$}

This is the most complicated case considered in the present paper. The first $S_{6}=$ 42 terms are those connected with $N=6$ because the time point 6 is now a free point of time on the scale; see Figure 9 and a list of terms below (69). The energy terms can be constructed by substituting the product

$$
P V
$$

at the end of any main bracket expression entering the energy term belonging to $N=6$ obtained in Section 6: 


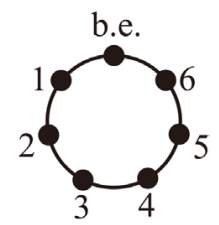

Figure 9. Time scale for the perturbation order $N=7$. Beyond the beginning-end (b.e.) point, six other points $(1,2,3,4,5$ and 6$)$ are present on the scale.

$\langle V P V P V P V P V P V P V\rangle-\left\langle V P^{2} V P V P V P V P V\right\rangle \Delta E_{1}-\left\langle V P^{2} V P V P V P V\right\rangle \Delta E_{2}$ $-\left\langle V P^{2} V P V P V P V\right\rangle \Delta E_{1}+\left\langle V P^{3} V P V P V P V\right\rangle\left(\Delta E_{1}\right)^{2}-\left\langle V P^{2} V P V P V\right\rangle \Delta E_{3}$ $+\left\langle V P^{3} V P V P V\right\rangle \Delta E_{1} \Delta E_{2}+\left\langle V P^{3} V P V P V\right\rangle \Delta E_{2} \Delta E_{1}-\left\langle V P^{4} V P V P V\right\rangle\left(\Delta E_{1}\right)^{3}$ $-\left\langle V P V P^{2} V P V P V\right\rangle \Delta E_{2}+\left\langle V P V P^{3} V P V P V\right\rangle\left(\Delta E_{1}\right)^{2}-\left\langle V P V P V P^{2} V P V P V\right\rangle \Delta E_{1}$ $+\left\langle V P^{2} V P^{2} V P V P V\right\rangle\left(\Delta E_{1}\right)^{2}-\left\langle V P^{2} V P V\right\rangle \Delta E_{4}+\left\langle V P^{3} V P V\right\rangle \Delta E_{1} \Delta E_{3}$ $+\left\langle V P^{3} V P V\right\rangle\left(\Delta E_{2}\right)^{2}+\left\langle V P^{3} V P V\right\rangle \Delta E_{3} \Delta E_{1}-\left\langle V P^{4} V P V\right\rangle\left(\Delta E_{1}\right)^{2} \Delta E_{2}$ $-\left\langle V P^{4} V P V\right\rangle \Delta E_{1} \Delta E_{2} \Delta E_{1}-\left\langle V P^{4} V P V\right\rangle \Delta E_{2}\left(\Delta E_{1}\right)^{2}+\left\langle V P^{5} V P V\right\rangle\left(\Delta E_{1}\right)^{4}$ $-\left\langle V P V P^{2} V P V\right\rangle \Delta E_{3}+\left\langle V P V P^{3} V P V\right\rangle \Delta E_{1} \Delta E_{2}+\left\langle V P V P^{3} V P V\right\rangle \Delta E_{2} \Delta E_{1}$ $-\left\langle V P V P^{4} V P V\right\rangle\left(\Delta E_{1}\right)^{3}-\left\langle V P V P V P^{2} V P V\right\rangle \Delta E_{2}+\left\langle V P V P V P^{3} V P V\right\rangle\left(\Delta E_{1}\right)^{2}$ $+\left\langle V P^{2} V P^{2} V P V\right\rangle \Delta E_{1} \Delta E_{2}-\left\langle V P^{2} V P^{3} V P V\right\rangle\left(\Delta E_{1}\right)^{3}-\left\langle V P V P V P V P^{2} V P V\right\rangle \Delta E_{1}$ $+\left\langle V P^{2} V P V P^{2} V P V\right\rangle\left(\Delta E_{1}\right)^{2}+\left\langle V P^{2} V P^{2} V P V\right\rangle \Delta E_{2} \Delta E_{1}-\left\langle V P^{3} V P^{2} V P V\right\rangle\left(\Delta E_{1}\right)^{3}$ $+\left\langle V P V P^{2} V P^{2} V P V\right\rangle\left(\Delta E_{1}\right)^{2}$.

The next $S_{6}=42$ energy terms come from contractions of point 6 with point 1 and the points between 1 and 6 :

$$
\begin{aligned}
& 1: 6 \rightarrow-\left\langle V P^{2} V\right\rangle \Delta E_{5} \\
& 1: 2: 6 \rightarrow\left\langle V P^{3} V\right\rangle \Delta E_{1} \Delta E_{4} \\
& 1: 3: 6 \rightarrow\left\langle V P^{3} V\right\rangle \Delta E_{2} \Delta E_{3} \\
& 1: 4: 6 \rightarrow\left\langle V P^{3} V\right\rangle \Delta E_{3} \Delta E_{2} \\
& 1: 5: 6 \rightarrow\left\langle V P^{3} V\right\rangle \Delta E_{4} \Delta E_{1} \\
& 1: 2: 3: 6 \rightarrow-\left\langle V P^{4} V\right\rangle\left(\Delta E_{1}\right)^{2} \Delta E_{3} \\
& 1: 2: 4: 6 \rightarrow-\left\langle V P^{4} V\right\rangle \Delta E_{1}\left(\Delta E_{2}\right)^{2} \\
& 1: 2: 5: 6 \rightarrow-\left\langle V P^{4} V\right\rangle \Delta E_{1} \Delta E_{3} \Delta E_{1} \\
& 1: 3: 4: 6 \rightarrow-\left\langle V P^{4} V\right\rangle \Delta E_{2} \Delta E_{1} \Delta E_{2} \\
& 1: 3: 5: 6 \rightarrow-\left\langle V P^{4} V\right\rangle\left(\Delta E_{2}\right)^{2} \Delta E_{1} \\
& 1: 4: 5: 6 \rightarrow-\left\langle V P^{4} V\right\rangle \Delta E_{3}\left(\Delta E_{1}\right)^{2}
\end{aligned}
$$




$$
\begin{aligned}
& 1: 2: 3: 4: 6 \rightarrow\left\langle V P^{5} V\right\rangle\left(\Delta E_{1}\right)^{3} \Delta E_{2} \\
& 1: 2: 3: 5: 6 \rightarrow\left\langle V P^{5} V\right\rangle\left(\Delta E_{1}\right)^{2} \Delta E_{2} \Delta E_{1} \\
& 1: 2: 4: 5: 6 \rightarrow\left\langle V P^{5} V\right\rangle \Delta E_{1} \Delta E_{2}\left(\Delta E_{1}\right)^{2} \\
& 1: 3: 4: 5: 6 \rightarrow\left\langle V P^{5} V\right\rangle \Delta E_{2}\left(\Delta E_{1}\right)^{3} \\
& 1: 2: 3: 4: 5: 6 \rightarrow-\left\langle V P^{6} V\right\rangle\left(\Delta E_{1}\right)^{5}
\end{aligned}
$$

which give also a set of $S_{6}=42$ perturbation terms:

$$
14+5+2+2+5+2+1+2+1+1+2+1+1+1+1+1=42
$$

because a maximum of 6 points coupled together. Here (87) is a sum of the number of the perturbation terms indicated in brackets at the end of each row in (71)-(86).

The next contractions of the time points give $S_{5}=14$ terms because of a maximal number of 5 points entering contractions:

$$
\begin{aligned}
& 2: 6 \rightarrow-\left\langle V P V P^{2} V\right\rangle \Delta E_{4} \\
& 2: 3: 6 \rightarrow\left\langle V P V P^{3} V\right\rangle \Delta E_{1} \Delta E_{3} \\
& 2: 4: 6 \rightarrow\left\langle V P V P^{3} V\right\rangle\left(\Delta E_{2}\right)^{2} \\
& 2: 5: 6 \rightarrow\left\langle V P V P^{3} V\right\rangle \Delta E_{3} \Delta E_{1} \\
& 2: 3: 4: 6 \rightarrow-\left\langle V P V P^{4} V\right\rangle\left(\Delta E_{1}\right)^{2} \Delta E_{2} \\
& 2: 3: 5: 6 \rightarrow-\left\langle V P V P^{4} V\right\rangle \Delta E_{1} \Delta E_{2} \Delta E_{1} \\
& 2: 4: 5: 6 \rightarrow-\left\langle V P V P^{4} V\right\rangle \Delta E_{2}\left(\Delta E_{1}\right)^{2} \\
& 2: 3: 4: 5: 6 \rightarrow\left\langle V P V P^{5} V\right\rangle\left(\Delta E_{1}\right)^{4}
\end{aligned}
$$

Next come $S_{4}=5$ energy terms due to contractions

$$
\begin{aligned}
& 3: 6 \rightarrow-\left\langle V P V P V P^{2} V\right\rangle \Delta E_{3} \\
& 3: 4: 6 \rightarrow\left\langle V P V P V P^{3} V\right\rangle \Delta E_{1} \Delta E_{2} \\
& 3: 5: 6 \rightarrow\left\langle V P V P V P^{3} V\right\rangle \Delta E_{2} \Delta E_{1} \\
& 3: 4: 5: 6 \rightarrow-\left\langle V P V P V P^{4} V\right\rangle\left(\Delta E_{1}\right)^{3}
\end{aligned}
$$

which do exist with free time points 1 and 2 on the scale giving the energy terms presented above. But also we can have contractions combined with 1:2 giving other $S_{4}=5$ energy terms:

$$
\begin{aligned}
& 1: 2 \cap 3: 6 \rightarrow\left\langle V P^{2} V P^{2} V\right\rangle \Delta E_{1} \Delta E_{3} \\
& 1: 2 \cap 3: 4: 6 \rightarrow-\left\langle V P^{2} V P^{3} V\right\rangle\left(\Delta E_{1}\right)^{2} \Delta E_{2} \\
& 1: 2 \cap 3: 5: 6 \rightarrow-\left\langle V P^{2} V P^{3} V\right\rangle \Delta E_{1} \Delta E_{2} \Delta E_{1}
\end{aligned}
$$




$$
1: 2 \bigcap 3: 4: 5: 6 \rightarrow\left\langle V P^{2} V P^{4} V\right\rangle\left(\Delta E_{1}\right)^{4}
$$

Let us note that each $\Delta E_{3}$ in (96) and (100) gives $S_{3}=2$ terms.

Another set of contractions involving point 6 represents

$$
\begin{aligned}
& 4: 6 \rightarrow-\left\langle V P V P V P V P^{2} V\right\rangle \Delta E_{2} \\
& 4: 5: 6 \rightarrow\left\langle V P V P V P V P^{3} V\right\rangle\left(\Delta E_{1}\right)^{2}
\end{aligned}
$$

on condition the time points 1, 2, and 3 are left free. But (104) and (105) can be combined also with contractions of the points 1,2, and 3 giving

$$
\begin{aligned}
& 1: 2 \bigcap 4: 6 \rightarrow\left\langle V P^{2} V P V P^{2} V\right\rangle \Delta E_{1} \Delta E_{2}, \\
& 1: 2 \bigcap 4: 5: 6 \rightarrow-\left\langle V P^{2} V P V P^{3} V\right\rangle\left(\Delta E_{1}\right)^{3}, \\
& 1: 3 \bigcap 4: 6 \rightarrow\left\langle V P^{2} V P^{2} V\right\rangle\left(\Delta E_{2}\right)^{2}, \\
& 1: 3 \bigcap 4: 5: 6 \rightarrow-\left\langle V P^{2} V P^{3} V\right\rangle \Delta E_{2}\left(\Delta E_{1}\right)^{2}, \\
& 1: 2: 3 \cap 4: 6 \rightarrow-\left\langle V P^{3} V P^{2} V\right\rangle\left(\Delta E_{1}\right)^{2} \Delta E_{2}, \\
& 1: 2: 3 \cap 4: 5: 6 \rightarrow\left\langle V P^{3} V P^{3} V\right\rangle\left(\Delta E_{1}\right)^{4}, \\
& 2: 3 \cap 4: 6 \rightarrow\left\langle V P V P^{2} V P^{2} V\right\rangle \Delta E_{1} \Delta E_{2}, \\
& 2: 3 \cap 4: 5: 6 \rightarrow-\left\langle V P V P^{2} V P^{3} V\right\rangle\left(\Delta E_{1}\right)^{3} .
\end{aligned}
$$

In effect the number of terms due to (104)-(113) is equal to $2 S_{4}=10$ because the points 1, 2 and 3 can combine in $S_{4}=5$ ways.

The last set of contractions containing point 6 is represented by $5: 6$. When a combination of 5:6 with the set of free time points $1,2,3$, and 4 is considered we obtain

$$
5: 6 \rightarrow-\left\langle V P V P V P V P V P^{2} V\right\rangle \Delta E_{1}
$$

The remaining combinations with 5:6 are due to contractions between points 1 , 2, 3 and 4:

$$
\begin{aligned}
& 1: 2 \bigcap 5: 6 \rightarrow\left\langle V P^{2} V P V P V P^{2} V\right\rangle\left(\Delta E_{1}\right)^{2} \\
& 1: 3 \bigcap 5: 6 \rightarrow\left\langle V P^{2} V P V P^{2} V\right\rangle \Delta E_{2} \Delta E_{1} \\
& 1: 4 \bigcap 5: 6 \rightarrow\left\langle V P^{2} V P^{2} V\right\rangle \Delta E_{3} \Delta E_{1} \\
& 1: 2: 3 \cap 5: 6 \rightarrow-\left\langle V P^{3} V P V P^{2} V\right\rangle\left(\Delta E_{1}\right)^{3} \\
& 1: 2: 4 \cap 5: 6 \rightarrow-\left\langle V P^{3} V P^{2} V\right\rangle \Delta E_{1} \Delta E_{2} \Delta E_{1} \\
& 1: 3: 4 \cap 5: 6 \rightarrow-\left\langle V P^{3} V P^{2} V\right\rangle \Delta E_{2}\left(\Delta E_{1}\right)^{2} \\
& 1: 2: 3: 4 \cap 5: 6 \rightarrow\left\langle V P^{4} V P^{2} V\right\rangle\left(\Delta E_{1}\right)^{4} \\
& 1: 2 \bigcap 3: 4 \cap 5: 6 \rightarrow-\left\langle V P^{2} V P^{2} V P^{2} V\right\rangle\left(\Delta E_{1}\right)^{3}
\end{aligned}
$$




$$
\begin{aligned}
& 2: 3 \cap 5: 6 \rightarrow\left\langle V P V P^{2} V P V P^{2} V\right\rangle\left(\Delta E_{1}\right)^{2} \\
& 2: 4 \bigcap 5: 6 \rightarrow\left\langle V P V P^{2} V P^{2} V\right\rangle \Delta E_{2} \Delta E_{1} \\
& 2: 3: 4 \cap 5: 6 \rightarrow-\left\langle V P V P^{3} V P^{2} V\right\rangle\left(\Delta E_{1}\right)^{3} \\
& 3: 4 \cap 5: 6 \rightarrow\left\langle V P V P V P^{2} V P^{2} V\right\rangle\left(\Delta E_{1}\right)^{2}
\end{aligned}
$$

The total number of energy terms due to (114)-(126) is $S_{5}=14$ which is the number of combinations due to presence of the 4 free time points, see (8) and Table 1 .

In total we obtain for $N=7$ the $S_{6}=42$ energy terms collected in (70), next also $S_{6}=42$ energy terms collected in (87). Another set of terms is given in the formulae from (88) to (95) which provide us with

$$
5+2+1+2+1+1+1+1=14
$$

terms. The next $2 S_{8}=10$ perturbation terms are given by the formulae (96)-(99) and (100)-(103), but also 10 terms are provided by (104)-(113). Finally contraction 5:6 gives from (114) to (126) the energy terms whose number is

$$
1+1+1+2+1+1+1+1+1+1+1+1+1=14=S_{5} .
$$

This makes a total number of kinds of the energy perturbation terms belonging to $N=7$ equal to:

$$
42+42+14+10+10+14=132=S_{7}
$$

which is not only in accordance with the formula (8), but satisfies also the formula:

$$
S_{1} S_{6}+S_{2} S_{5}+S_{3} S_{4}+S_{4} S_{3}+S_{5} S_{2}+S_{6} S_{1}=S_{7}
$$

The result in (130) is a special case of a general formula which holds for calculating $S_{N}$ :

$$
S_{N}=S_{1} S_{N-1}+S_{2} S_{N-2}+S_{3} S_{N-3}+\cdots+S_{N-3} S_{3}+S_{N-2} S_{2}+S_{N-1} S_{1} .
$$

\section{General Characteristics of the Energy Perturbation Terms}

In general the terms of the Schrödinger perturbation energy which originate from a non-degenerate quantum state are represented by the products of the contribution due to the main loop of time and contributions due to the side loops. This second kind of contributions is equal to definite perturbation energies

$$
\Delta E^{\left(N^{\prime}\right)}
$$

of the order $N^{\prime}$ smaller than the examined order $N$. The formulae of the kind of (132) which are due to the side loops of time provide an important simplification of the perturbed energy calculations.

The side loops originate from contractions of the time points on the main loop characteristic for a given perturbation order $N$. In effect the order $N^{\prime}$ characteristic for any expression (132) is equal to a difference of the indices representing the time points entering contraction. There can exist also multiple 
contractions giving products

$$
\Delta E^{\left(N^{\prime}\right)} \Delta E^{\left(N^{\prime \prime}\right)} \Delta E^{\left(N^{\prime \prime \prime}\right)} \cdots
$$

where $N^{\prime}, N^{\prime \prime}, N^{\prime \prime \prime}, \cdots$ are defined by the difference between the time point indices participating successively in a multiple contraction.

One point on the main loop, which is the beginning-end point of the scale, is excluded from contractions. Respectively, for each $N$ does exist only one energy term which is given solely by the main loop of time; this loop of time has no contractions and the corresponding energy term does not involve the contributions of the side loops.

The kind of energy contributions due to the main loop is in general different than that given in (132) or (133). In the absence of contractions the main loop gives a single energy term equal to

$$
\langle V P V P V P \cdots P V P V\rangle
$$

in which the number of $V$ is equal to the perturbation order $N$ and number of $P$ amounts $N-1$. In effect any time point on the scale-excepting the beginning-end point-has its own $P$, and the number of terms $V$ is equal to the number of distances separating the neighbouring time points on the scale.

The contractions change the power exponents of $P$ entering (134) which are all equal to 1 in (134) into the exponents

$$
\tau>1
$$

The number $\tau$ is equal to the number of the time points participating in contraction, therefore $\tau$ becomes equal to the number of the side loops created by contraction increased by one. So for one side loop present in a given time point

$$
\tau=1+1,
$$

for two side loops present in the same point

$$
\tau=1+2,
$$

etc. In effect the number of $P$ terms which remain on the main loop of time and have the exponents represented by (135) is equal to the number of the time contractions present on the scale.

An important feature is that some $P$ entering (134) can be shifted to the side loops. This situation holds in case when the differences between the time-point indices entering contraction are larger than 1 . For example the contraction between the points 1 and 3 shifts the point 2 -and its $P$ term-to a side loop. This is an expected result if we note that $N^{\prime}, N^{\prime \prime}, N^{\prime \prime \prime}, \cdots$ in (132) or (133) can be larger than unity.

Nevertheless the sum of the power exponents of $P$ present along the scale-those which remain on the main loop as well as those which are shifted to the side loop or loops-should remain unchanged. In effect any perturbation term belonging to a given $N$ has the same number $N-1$ of the $P$ terms and number $N$ of the $V$ terms, because the total number of $V$ terms in the main loop and side loops remains constant. All $S_{N}$ terms give different diagrams along the 
time scale plotted for a given $N$, but the computational results due to several diagrams can be equal which is the effect of the diagram symmetry.

\section{General View on Contractions of the Time Points on the Time Scales and Their Application}

The way of calculating the Schrödinger perturbation energy-called sometimes also the Rayleigh-Schrödinger perturbation series-presented in the paper is rather different than procedures applied in the former approaches; see e.g. [12]. In fact the terms entering the series can be obtained mainly from an analysis of the geometry of the time-point patterns present on the scale, and by applying the rules connecting that geometry with the time-independent matrix elements entering the Schrödinger wave mechanics, than by proceeding according to any of the developed wave-mechanical perturbation formalisms.

A basic property concerns the importance of contraction points of time entering the applied time scale and their origin. At the first step-i.e. in the absence of contractions-the scale giving the perturbation energy of order $N$ is assumed to have $N$ separate time points on it, and one of these points is the beginning-end point of the scale. Evidently for $N=1$ there is present only the beginning-end point. We assume that this point cannot be submitted to contractions in case of any $N$. Therefore an increase of the perturbation order to $N=2$ gives the scale which has two points on it: one is the beginning-end point and the second point is allowed which remains free.

A substantial difference in the calculation scheme begins with $N=3$. In this case there exist outside the beginning-end point two other points which (a) can be left separated each from other, but also (b) can be contracted together. What does it mean from the point of view of the scale geometry and the calculations? The case (a) represents two separate points of time any of which gives its own contribution to the perturbation energy term belonging to $N=3$. According to Section 6 the energy term supplied by these both points together becomes

$$
\langle V P V P V\rangle \text {. }
$$

So what is the effect of contraction of two points on the scale upon the perturbation energy? Geometrically it means that contraction of point 1 with point 2 labelled by the symbol

$$
1: 2
$$

produces two different scales of time. The first one is the scale containing the beginning-end point of the original loop of time. The time parameters $t_{1}$ and $t_{2}$ labelling the perturbation events on that loop become equal, so an alternative formula to $(137)$ is

$$
t_{1}=t_{2} .
$$

This means that there is no possibility to have a time point between $t_{1}$ and $t_{2}$ on the main loop of time. But contraction represented by (137a) implies creation of a supplementary loop of time for which the situation given in (137a) represents the beginning-end point of time. This supplementary loop, called also a side 
loop, is independent of the original (main) loop of time. The side loop has no time points on it excepting the beginning-end point (137a). Such loop is identical with the main loop of time for $N=1$; see Figure 2 .

In effect of creation of the new loop of time, a new-i.e. the second-perturbation term of energy is obtained for the perturbation order $N=3$ beyond the term given in (136). This is a product of two bracket terms, viz.

$$
-\left\langle V P^{2} V\right\rangle\langle V\rangle=-\left\langle V P^{2} V\right\rangle \Delta E_{1}
$$

The first bracket term is given in (21), the second term is simply the perturbation energy of order one $(N=1)$; see (16).

For $N>3$, say for $N=4$, the free time points on the main loop can be $t_{1}, t_{2}$ and $t_{3}$, whereas the point $t_{4}$ is assumed to represent the beginning-end point on the loop; see Figure 6. In this case-beyond contractions between the neighbouring time points like (137) and (137a)—-the contraction between the non-neighbouring time points

$$
t_{1}=t_{3}
$$

or

$$
1: 3
$$

is also possible. This contraction implies that the point $t_{2}$ which originally is placed between $t_{1}$ and $t_{3}$ should be shifted to a side loop. This side loop has its beginning-end point given by contraction (139) or (139a), but one free time point, namely $t_{2}$, does remain on the loop. In effect the side loop becomes identical to that for $N=2$; see Figure 3. The perturbation energy due to contraction (139) is therefore equal to product

$$
-\left\langle V P^{2} V\right\rangle\langle V P V\rangle=-\left\langle V P^{2} V\right\rangle \Delta E_{2} .
$$

For the first bracket term in (140) see (21), for the second bracket term—see (17). The minus sign in (138) and (140) is dictated by the even number of the bracket terms entering product.

In Table 2 we summarize the data on the time points and their contractions which give the energy terms belonging to the perturbation orders from $N=1$ to $N=6$.

\section{Conclusions}

The history of investigations on time is probably as old as the history of science. In the Newtonian formulation of mechanics, the time interval is independent of any other physical parameter; in the theory of relativity, the dependence of the time interval is mainly due to the speed of the observed change.

But in many cases, including the problem considered in the present paper, the influence of the speed effect-or other physical parameters-on the time intervals can be neglected. The kind of approach proposed here is different than the Newtonian-like, namely an insight into time given by Leibniz [13] [14] [15] seems to be here of importance. 
Table 2. Number of the time points on the main loop of time and symbols of contraction points giving the side loops for the perturbation orders from $N=1$ to $N=6$. The second component entering the last column is equal to the contractions number of the time points (see the 3 rd column).

\begin{tabular}{|c|c|c|c|}
\hline$N$ & $\begin{array}{l}\text { Number of the time } \\
\text { points on the main } \\
\text { loop of time }\end{array}$ & Contractions of the time points & $\begin{array}{c}\text { Total number of the } \\
\text { perturbation terms } \\
S_{N}(\text { see Table } 1)\end{array}$ \\
\hline 1 & $1+0$ & no contractions & 1 \\
\hline 2 & $1+1$ & no contractions & 1 \\
\hline 3 & $1+2$ & $1: 2$ & $1+1=2$ \\
\hline 4 & $1+3$ & $\begin{array}{c}1: 2,1: 3,2: 3 \\
1: 2: 3\end{array}$ & $1+4=5$ \\
\hline 5 & $1+4$ & $\begin{array}{c}1: 2,1: 3,1: 4 \\
2: 3,2: 4,3: 4 \\
1: 2 \cap 3: 4,1: 2: 3 \\
1: 2: 4,1: 3: 4,1: 4 \bigcap 2: 3 \\
1: 2: 3: 4, \quad 2: 3: 4\end{array}$ & $1+13=14$ \\
\hline 6 & $1+5$ & $\begin{array}{c}1: 2,1: 3,1: 4,1: 5,2: 3, \\
2: 4,2: 5,3: 4,3: 5,4: 5, \\
1: 2: 3,1: 2: 4,1: 2: 5, \\
1: 3: 4,1: 3: 5,1: 4: 5, \\
2: 3: 4,2: 3: 5,2: 4: 5, \\
3: 4: 5,1: 2: 3: 4,1: 2: 3: 5, \\
1: 2: 4: 5,1: 3: 4: 5,2: 3: 4: 5 \\
1: 2: 3: 4: 5,1: 2: 3 \cap 4: 5,1: 2 \cap 3: 5,1: 2 \cap 4: 5, \\
1: 2 \cap 3: 4: 5,1: 2 \cap 3: 4,1: 3 \cap 4: 5,2: 3 \cap 4: 5, \\
1: 4 \cap 2: 3,1: 5 \cap 2: 4,1: 4: 5 \cap 2: 3,1: 5 \cap 2: 3, \\
1: 5 \cap 3: 4,1: 5 \cap 2: 3: 4,1: 2: 5 \cap 3: 4,2: 5 \cap 3: 4\end{array}$ & $1+41=42$ \\
\hline
\end{tabular}

Leibniz idea was that time is represented by a sequence of events which appear successively in a definite order. A knowledge of the sizes of time intervals between the separate events become then of not much use, since the main point concerns an arrangement of the events along a proper scale of time.

In the present paper the problem of the shape of the time scale and its physical verification has been attached to the Schrödinger perturbation theory. Physically this means that the history of a perturbed quantum state-done by a potential independent of time-becomes of importance. This history has a non-relativistic background, for it refers to a general applicability of the non-relativistic Schrödinger equation in the quantum physics. 
In principle the perturbation theory-linked also with the Schrödinger's authorship [3] - provides us with a method how solutions known for a simple problem can provide us with an approximate knowledge of more complicated Schrödinger's solutions. A difficulty was that a tedious procedure had to be applied in order to extract to calculations the separate kinds of energy terms belonging to a large perturbation order $N$. This difficulty could be much reduced when the time scale of a circular character composed of the $N$ collision time points of a quantum system with the perturbation potential $V^{\text {per }}$ is assumed for each $N$.

The number of the allowed time-point arrangements on the scale provides us precisely with the $S_{N}$ perturbation energy terms characteristic for a given $N$. In effect the perturbation terms should not be derived with the aid of a usually tedious iterative procedure connected with solving the perturbed Schrödinger equation, but can be readily obtained by analyzing the contractions to which the time points are submitted along the scale.

It should be noted that agreement of the results for $S_{N}$, as well as the energy perturbation terms for a given $N$, obtained in the present theory with those calculated by the Schrödinger formalism is not proved in general but has been demonstrated in the paper for the perturbation orders beginning from $N=1$ to $N=7$.

No time intervals or continuous time variables are considered in the paper. The calculations are based on definite sets of the discrete time points representing the collisions of an unperturbed non-degenerate quantum system with a time-independent perturbation potential.

\section{Conflicts of Interest}

The author declares no conflicts of interest regarding the publication of this paper.

\section{References}

[1] Berliner, A. and Scheel, K. (1932) Physikalisches Handwörterbuch. Springer, Berlin. https://doi.org/10.1007/978-3-642-99643-6

[2] Landau, L.D. and Lifshitz, E.M. (1948) Field Theory. 2nd Edition, OGIZ, Moscow. (In Russian)

[3] Schrödinger, E. (1926) Annalen der Physik, 80, 437.

[4] Schiff, L.I. (1968) Quantum Mechanics. 3rd Edition, McGraw-Hill, New York.

[5] Huby, R. (1961) Proceedings of the Physical Society (London), 78, 529. https://doi.org/10.1088/0370-1328/78/4/306

[6] Tong, B.Y. (1962) Proceedings of the Physical Society (London), 80, 1101. https://doi.org/10.1088/0370-1328/80/5/308

[7] Feynman, R.P. (1966) Science, 153, 699-708. https://doi.org/10.1126/science.153.3737.699

[8] Mattuck, R.D. (1976) A Guide to Feynman Diagrams of the Many-Body Problem. 2nd Edition, McGraw-Hill, New York. 
[9] Olszewski, S. (1991) Zeitschrift fuer Naturforschung, 46A, 313-320. https://doi.org/10.1515/zna-1991-0404

[10] Olszewski, S. (2004) Studia Philosophiae Christianae, 40, 57.

[11] Olszewski, S. (2018) Journal of Modern Physics, 9, 1491-1521. https://doi.org/10.4236/jmp.2018.98093

[12] Ziman, J.M. (1969) Elements of Advanced Quantum Theory. Cambridge University Press, Cambridge.

[13] Leibniz, G.W. (1924) Hauptschriften zur Grundlagen der Philosophie. Vol. 1, Leipzig.

[14] Rescher, N. (2013) On Leibniz. University of Pittsburgh Press, Pittsburgh. https://doi.org/10.2307/j.ctt7zw8g2

[15] Szumilewicz, I. (1964) On the Direction of the Time Flow. Państwowe Wydawnictwo Naukowe, Warszawa. (In Polish) 